\title{
Article \\ Comparison of Peritoneal Carcinomatosis Scoring Methods in Predicting Resectability and Prognosis in Gynecologic Malignancies
}

\author{
María Teresa Climent ${ }^{1,2, * \mathbb{D}}$, Anna Serra ${ }^{1,2} \mathbb{D}$, Juan Gilabert-Estellés ${ }^{2,3}$, Juan Gilabert-Aguilar ${ }^{2}$ \\ and Antoni Llueca ${ }^{1,2}$ (D) \\ 1 Department of Obstetrics and Gynecology, University General Hospital of Castellón, 12004 Castellón, Spain; \\ serraa@uji.es (A.S.); llueca@uji.es (A.L.) \\ 2 Department of Medicine, University of Jaume I, 12071 Castellón, Spain; juangilaeste@yahoo.es (J.G.-E.); \\ juangilabertaguilar@gmail.com (J.G.-A.) \\ 3 Department of Obstetrics and Gynecology, University General Hospital of Valencia, 46014 Valencia, Spain \\ * Correspondence: climarma@gmail.com
}

check for

updates

Citation: Climent, M.T.; Serra, A.; Gilabert-Estellés, J.; Gilabert-Aguilar, J.; Llueca, A. Comparison of

Peritoneal Carcinomatosis Scoring Methods in Predicting Resectability and Prognosis in Gynecologic Malignancies. J. Clin. Med. 2021, 10, 2553. https://doi.org/10.3390/jcm 10122553

Academic Editor: Michael Eichbaum

Received: 8 April 2021

Accepted: 3 June 2021

Published: 9 June 2021

Publisher's Note: MDPI stays neutral with regard to jurisdictional claims in published maps and institutional affiliations.

Copyright: (c) 2021 by the authors. Licensee MDPI, Basel, Switzerland. This article is an open access article distributed under the terms and conditions of the Creative Commons Attribution (CC BY) license (https:/ / creativecommons.org/licenses/by/ $4.0 /)$.
Abstract: Objective: Peritoneal carcinomatosis is a disease's presentation in the advanced stages of many gynecologic tumours. The distribution and volume of the disease are the main factors in achieving complete debulking. Diagnostic laparoscopy is a technique to allow evaluation of the disease. This study's objective is to compare two laparoscopic scores (Fagotti's index and Sugarbaker's peritoneal cancer index (PCI)) and assess the diagnostic accuracy to select patients for neoadjuvant treatment and reduce unnecessary laparotomies. Methods: A non-randomised retrospective cohort study was conducted in patients with peritoneal carcinomatosis (ovarian and endometrial origin) who underwent laparoscopy and subsequent laparotomy. We evaluated the scores' ability to predict incomplete surgery and whether they were related to the patients' prognosis. Results: We included 34 patients, of which $23.5 \%$ received neoadjuvant chemotherapy. The rate of complete cytoreductive surgery was $79.4 \%$ ( $n=27$ patients). The highest sensitivity was obtained with a PCI value greater than 20. It was the best parameter to determine incomplete debulking. Survival curves were analysed according to the "cut off" established for each score, and statically significant differences were found using PCI with respect to Fagotti's Index. However, these differences were not found with Fagotti's score. Conclusion: The best diagnostic method to classify patients with peritoneal cancer is the PCI. It could be adapted to each surgical team because it allows identifying the "cut off point", which depends on incomplete surgery rate.

Keywords: laparoscopy; peritoneal carcinomatosis; PCI; Fagotti index; resecability

\section{Introduction}

Ovarian cancer is a significant cause of death from gynaecologic cancer, with $70-80 \%$ of cases diagnosed in advanced stages [1,2]. Complete primary debulking surgery is an independent survival factor $[1,2]$ because the tumour residue's size is inversely related to overall survival $[3,4]$.

The distribution and volume of the disease are the main factors in achieving complete debulking. The FIGO classification is not a useful tool to determine the disease's extent due to patients' disparity with the same stage.

Among the diagnostic methods, laparotomy continues to be the most accurate one to determine the burden of disease in patients. It is an invasive method of diagnosis with associated complications [1].

Diagnostic laparoscopy is a less-invasive evaluation alternative that reduces suboptimal surgery from $39 \%$ to $10 \%[2,5]$. This technique can improve optimal surgery rates, limiting unnecessary morbidity and reducing costs derived from unnecessary laparotomy $[6,7]$. 
The application of an evaluation method that allows quantifying the disease in patients with peritoneal carcinomatosis would improve the test's accuracy.

The use of scores is a method to compare results and determine in an objective way the treatment indicated for each patient; however, it is currently a challenge.

The use of laparoscopic scores could reduce unnecessary laparotomies for those patients to whom the possibility of complete cytoreduction is scarce or non-existent. Neoadjuvant therapy and interval surgery are presented as valid alternatives for these patients [2].

The most widely used laparoscopic predictive models evaluating ovarian cancer's extension are the peritoneal carcinomatosis index, described by Sugarbaker, and the Fagotti score [8].

Traditionally, gynecologist oncologists in Europe have used Fagotti's index to predict cytorreductive surgery in laparotomy for gynecologic malignancies [9-11].

This study aims to compare these scores, assessing the diagnostic accuracy to select patients for neoadjuvant treatment and reduce unnecessary laparotomies, morbidity and mortality derived from them, and correlation with the prognosis of these patients.

\section{Material and Methods}

\subsection{Patients}

115 patients with diagnosis of peritoneal carcinomatosis.

Patients were required to meet all the following eligibility criteria:

- $\quad$ Aged 18 years old or older.

- Diagnosis of peritoneal carcinomatosis.

- Written consent of surgery.

We excluded patients who did not have diagnosis of peritoneal carcinomatosis or did not give consent for data collection.

71 patients $(61.7 \%)$ had not undergone laparoscopy at diagnosis, so they were excluded from the study.

Of 54 patients on whom laparoscopy was performed to evaluate primary debulking, $10(18.5 \%)$ were referred for neoadjuvant treatment without subsequent laparotomy.

A total of 81 patients were referred for neoadjuvant treatment for reasons attributable to the patient (e.g., comorbidity, anaesthetic risk), to the disease (e.g., metastasis, number of intestinal resections) or, criteria of unresectability established by the Multidisciplinary Unit of Abdominal Pelvic Surgery (MUAPOS) (Radiologic criteria; lung metastasis, hepatic metastasis in three or more hepatic segments, severe hepatic pedicle involvement and progression after neoadjuvant chemotherapy) $[1,3,6]$.The comparison was made among patients where the laparoscopy score suggested the possibility of performing a successful debulking surgery, and subsequent laparotomy was performed within a maximum period of 10 days (34 patients) (Figure 1 ).

The absence of tumour disease was defined as complete surgery, not distinguishing between optimal (tumour residue less than $1 \mathrm{~cm}$ ) and suboptimal surgery (tumour residue greater than $1 \mathrm{~cm}$ ). We included these two types of surgery under the term 'incomplete cytoreductive surgery' [4].

The patients' preoperative evaluation consisted of a complete gynaecological examination, transvaginal ultrasound, tumour markers (Ca 125, CEA, Ca 19.9 and HE4), complete analysis, and thoracic-abdominopelvic computed tomography. Informed consent was obtained from all patients, and the ethics committee approved the study. 


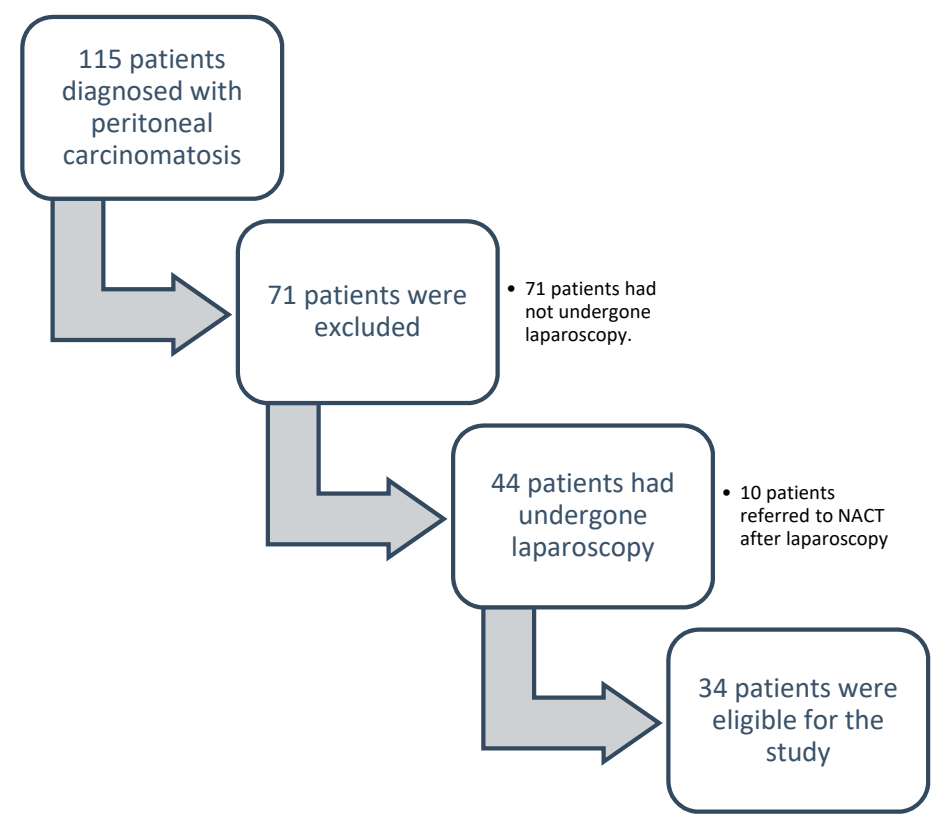

Figure 1. Patient selection.

\subsection{Surgical Technique}

A $15 \mathrm{~mm}$ supraumbilical longitudinal incision was made, and a $12 \mathrm{~mm}$ Hasson's trocar was introduced in the abdomen. A suprapubic $5 \mathrm{~mm}$ accessory trocar was placed, adding another trocar $5 \mathrm{~mm}$ in the iliac fossa if required, both under direct vision.

The entire abdominal cavity was examined, and a biopsy of the ovaries, metastatic nodules, or peritoneal surface was performed to confirm the diagnosis.

In our unit, it is considered that PCIs greater than 20 are associated with high morbidity with a decrease in the possibility of complete debulking. The value considered as unresectable in the case of the Fagotti index is higher than $8[12,13]$. However, the subjective evaluation of the oncologist surgeon, the histology of the disease, and the performance status of the patient are evaluated prior to the decision to perform surgery for posterior debulking.

If the patient is considered to be subject to debulking surgery, it was performed in the second stage, after histological diagnosis, with a xipho-pubic midline incision.

\subsection{Scores}

\subsubsection{Fagotti Score}

The Fagotti Score is based on the evaluation of seven parameters: omental cake, peritoneal carcinomatosis, diaphragmatic carcinomatosis, mesenteric retraction, stomach infiltration, bowel infiltration, and liver metastases. Each parameter is valued with a 0 if absent or 2 if present. The total value is between 0 and 14 . A value above or equal to 8 is related to suboptimal surgery $[7,12]$.

\subsubsection{Sugarbaker Score (PCI)}

The abdomen is divided into nine regions: central (0), right hypochondrium (1), left hypochondrium (3), epigastrium (2), left flank (4), left iliac fossa (5), pelvis (6), right iliac fossa (7), and right flank (8). Four regions corresponding to the digestive tract are added: upper jejunum (9), lower jejunum (10), upper ileum (11), and lower ileum (12). Each area scores 0 if there is no evidence of a tumour; 1 with a tumour smaller than $0.5 \mathrm{~cm}, 2$ with a tumour up to $5 \mathrm{~cm}$, and 3 with a tumour larger than $5 \mathrm{~cm}$ or confluent.

The value obtained is between 0 and 39 .

The "cut off" established in the bibliography is between 10 and $20[5,6]$.

The description of the scores is described in Appendix A. 


\subsection{Statical Analysis}

The objective of this retrospective descriptive non-randomised study is to compare the two laparoscopic scores to determine sensitivity, specificity, positive predictive value (PPV), negative predictive value (NPV), and diagnostic accuracy to predict suboptimal cytoreduction in patients with gynaecological peritoneal carcinomatosis.

The demographic characteristics of each patient, the tumour, and the surgical results obtained were analysed.

Parameters such as FIGO stage, PCI, and Fagotti score were determined for each patient who underwent laparoscopy and laparotomy.

The main objective was to determine if the models correlated with the incomplete debulking rate (optimal or suboptimal) and overall survival, using ROC (receiver operating characteristic) curves and the Kaplan-Meier method for survival.

Significance was assumed with a $p$-value less than 0.05 .

The statistical program used was IBM SPSS Statistics version 19 (IBM Corp, released in 2010. IBM SPSS Statistics for Windows, Version 19.0. Armonk, NY, USA).

\section{Results}

Between 2013 and 2017, 115 patients were diagnosed with peritoneal carcinomatosis at the University General Hospital of Castellón. The data were extracted from the database of the Multidisciplinary Unit of Abdominal Pelvic Surgery (MUAPOS). During the study period, 34 patients who underwent laparoscopy surgery and posterior cytoreductive surgery were included in the data analysis.

The mean age of the patients was 62 years.

According to the FIGO classification [1,2], 79.4\% ( $n=27$ patients) were classified as FIGO III, $20.6 \%$ ( $n=7$ patients) presented stage IV.

$58.8 \%$ were high-grade serous tumours.

The origin of the peritoneal carcinomatosis was ovarian in $85.3 \%$; in $14.7 \%$ of the patients, it was endometrial.

The mean tumour marker (Ca 125) was $1020.94 \mathrm{IU} / \mathrm{mL}$.

$23.5 \%$ of the patients received neoadjuvant chemotherapy.

Maneuvers were performed in the upper abdomen in $61.8 \%$ of the patients.

The complete cytoreduction rate was $79.4 \%$ ( $n=27$ patients) with $52.9 \%$ postoperative complications, of which $5.8 \%$ were severe.

The suboptimal cytoreduction rate was $20.6 \%$, leaving a macroscopic tumour residue equal or more than $1 \mathrm{~cm}$.

The clinicopathological features shown in the table (Table 1).

Overall survival at 5 years was 46.31 months (95\% CI 33.06-69.57) (Figure 2).

The mean of Fagotti's score with laparoscopic approach was 4.79 (se 0.406), and Sugarbaker's score was 13.03 (se 1.06).

A comparison of means between the two scores and their capacity to predict complete debulking applied by laparoscopy or laparotomy was conducted.

Statistically significant differences were found in the means of the Sugarbaker index in complete cytoreductive surgery (R0) about incomplete cytoreductive surgery (No R0) and not at all in the Fagotti's score (Tables 2 and 3). 
Table 1. Clinicopathological features.

\begin{tabular}{ccc}
\hline & $n=34$ & $\%$ \\
\hline Median age at surgery (years) & 61.97 & 23.5 \\
Neoadjuvant chemotherapy & 8 & 58.8 \\
Surgical procedures upper abdomen & 20 & 50 \\
Exitus & 17 & \\
Mean follow-up time (months) & 25.79 & 79.4 \\
Mean pre-surgical Ca 125 & 1020.94 & 85.3 \\
Complete cytoreductive surgery & 27 & 20.6 \\
Complete/optimal cytoreductive surgery & 29 & 79.4 \\
Suboptimal cytoreductive surgery & 7 & 20.6 \\
FIGO stage & & \\
III & 27 & 58.8 \\
IV & 7 & 20.6 \\
Histology & & 20.6 \\
Serous & 20 & 85.3 \\
Endometroid & 7 & 14.7 \\
Others & 7 & \\
Tumour origin & & 91.2 \\
Ovarian & 29 & 8.8 \\
Endometrial & 5 &
\end{tabular}

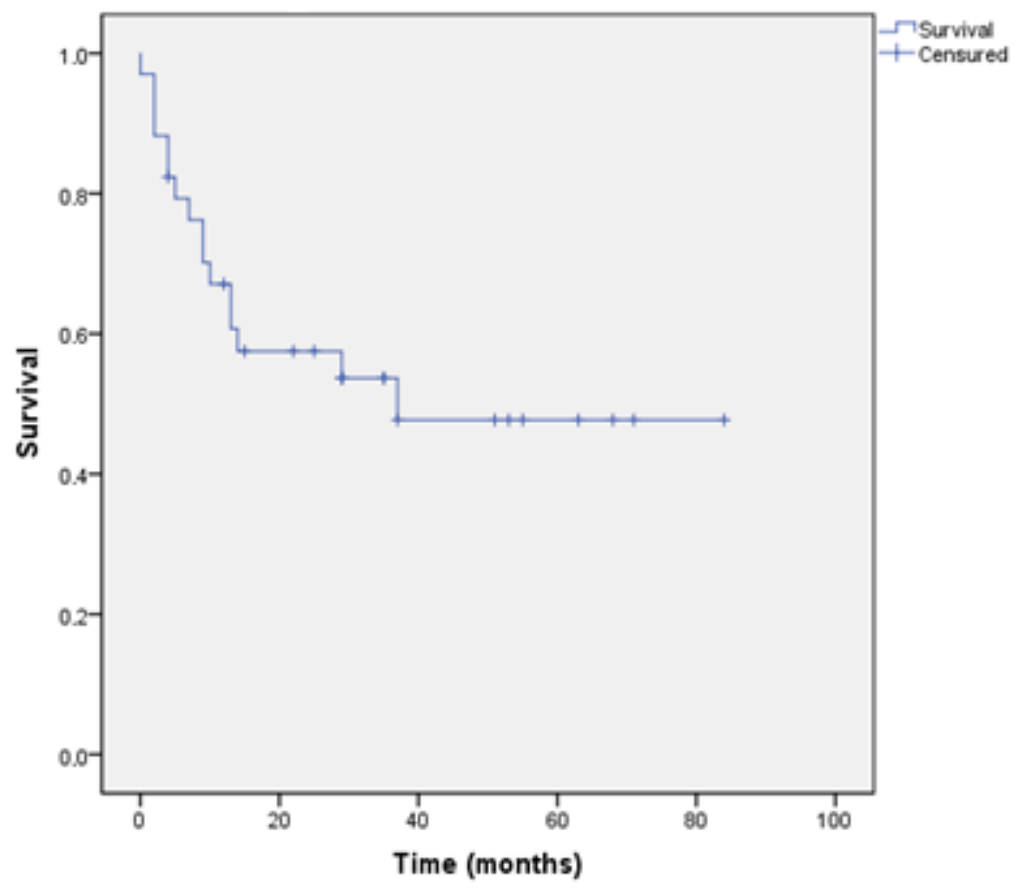

Figure 2. Overall Survival.

Table 2. Laparoscopic index and complete cytoreductive surgery.

\begin{tabular}{cccc}
\hline Laparoscopy & R0 & No R0 & $p$ \\
\hline PCI & $11.44($ se 1.13$)$ & $19.14($ se 1.16$)$ & 0.002 \\
Fagotti & $4.78(0.46)$ & $5.71($ se 0.808$)$ & 0.338 \\
\hline
\end{tabular}

R0: complete cytoreductive surgery. No R0: incomplete cytoreductive surgery. SE: standard error. 
Table 3. Laparotomic index and complete cytoreductive surgery.

\begin{tabular}{cccc}
\hline Laparotomy & R0 & No R0 & $p$ \\
\hline PCI & $10.30($ se 1.16) & $23.86($ se 1.43$)$ & 0.000 \\
Fagotti & $5.56($ se 0.59) & 7 (se 1.09) & 0.273 \\
\hline
\end{tabular}

R0: complete cytoreductive surgery. No R0: incomplete cytoreductive surgery.

The predictive capacity of the different indices applied laparoscopically to predict incomplete debulking surgery was evaluated.

The highest sensitivity was obtained with PCI value greater than 20, resulting in $43 \%$, with a specificity of $88 \%$, a PPV of $50 \%$ and an NPV of $86 \%$.

Based on the data provided, the Sugarbaker score was the best parameter to determine the possibility of incomplete debulking (Table 4).

Table 4. Diagnostic Parameters.

\begin{tabular}{cccccc}
\hline Laparoscopic Score & Sensibility & Specificity & PPV & NPV & Accuracy \\
\hline PCI $\geq 10$ & $30 \%$ & $88 \%$ & $30 \%$ & $100 \%$ & $53 \%$ \\
PCI $\geq 20$ & $43 \%$ & $88 \%$ & $50 \%$ & $86 \%$ & $79 \%$ \\
Fagotti $\geq 8$ & $14 \%$ & $81 \%$ & $16 \%$ & $78 \%$ & $68 \%$ \\
\hline
\end{tabular}

PPV: positive predictive value. NPV: negative predictive value.

The most significant discriminative capacity test was the peritoneal cancer index described by Sugarbaker, regardless of whether it was applied via laparoscopy or laparotomy (Figure 3).

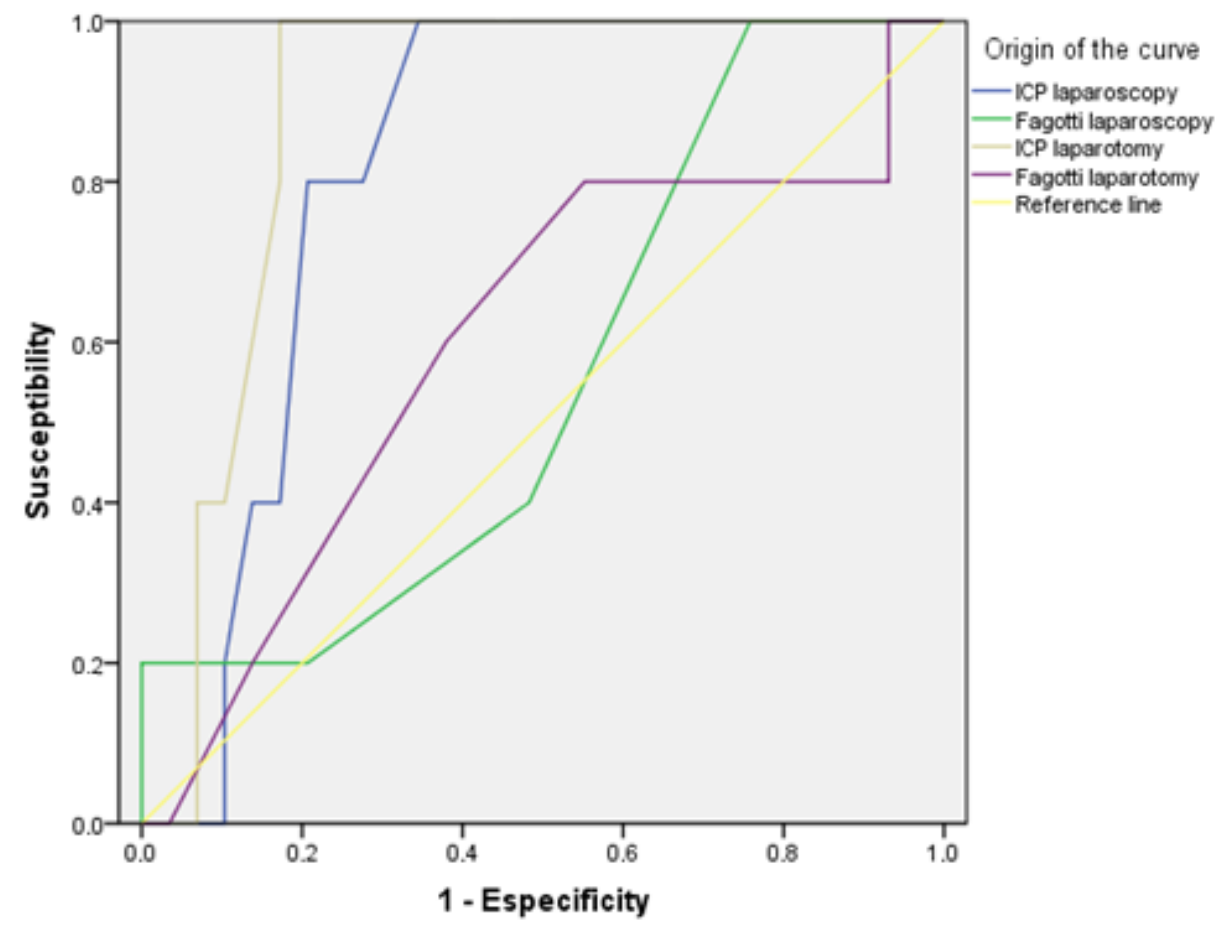

Figure 3. Roc curves shows the accuracy of these tests applied for laparotomy or laparoscopy.

This data was evaluated using the ROC curves.

Survival curves were analysed according to the "cut off" established for each score evaluated and determined if they had a prognostic impact when this score was applied via laparoscopy.

These tables show the overall survival, depending on whether the value of the index applied via laparoscopy is greater than the established values 10 and 20 for the Sugarbaker $\mathrm{PCI}$, and 8 for the Fagotti index. 
These differences in overall survival were found with statistically significant differences when using the PCI (Figures 4 and 5 and Tables 5 and 6); however, these differences were not found with the Fagotti's score (Figure 6 and Table 7).

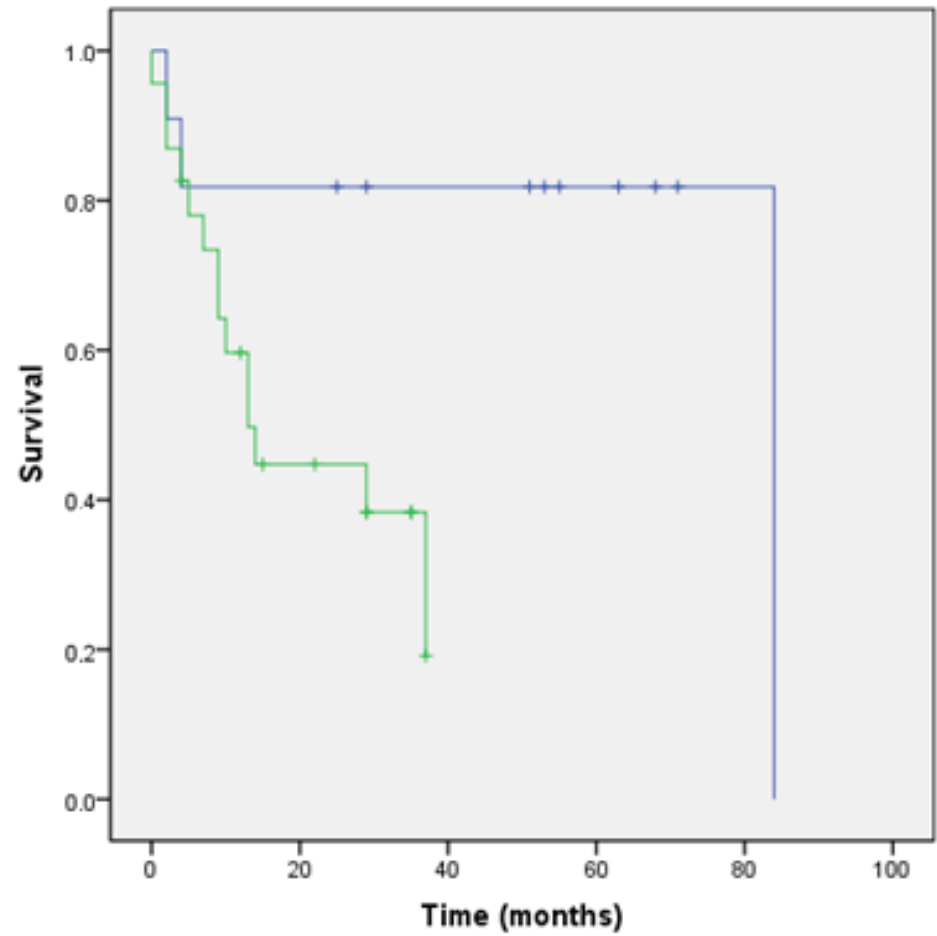
PCI Laparoscopy $>10$
$\pi<10$
$r>10$
$t<10$-censured
$+>10$-censured

Figure 4. Survival PCI > 10 .

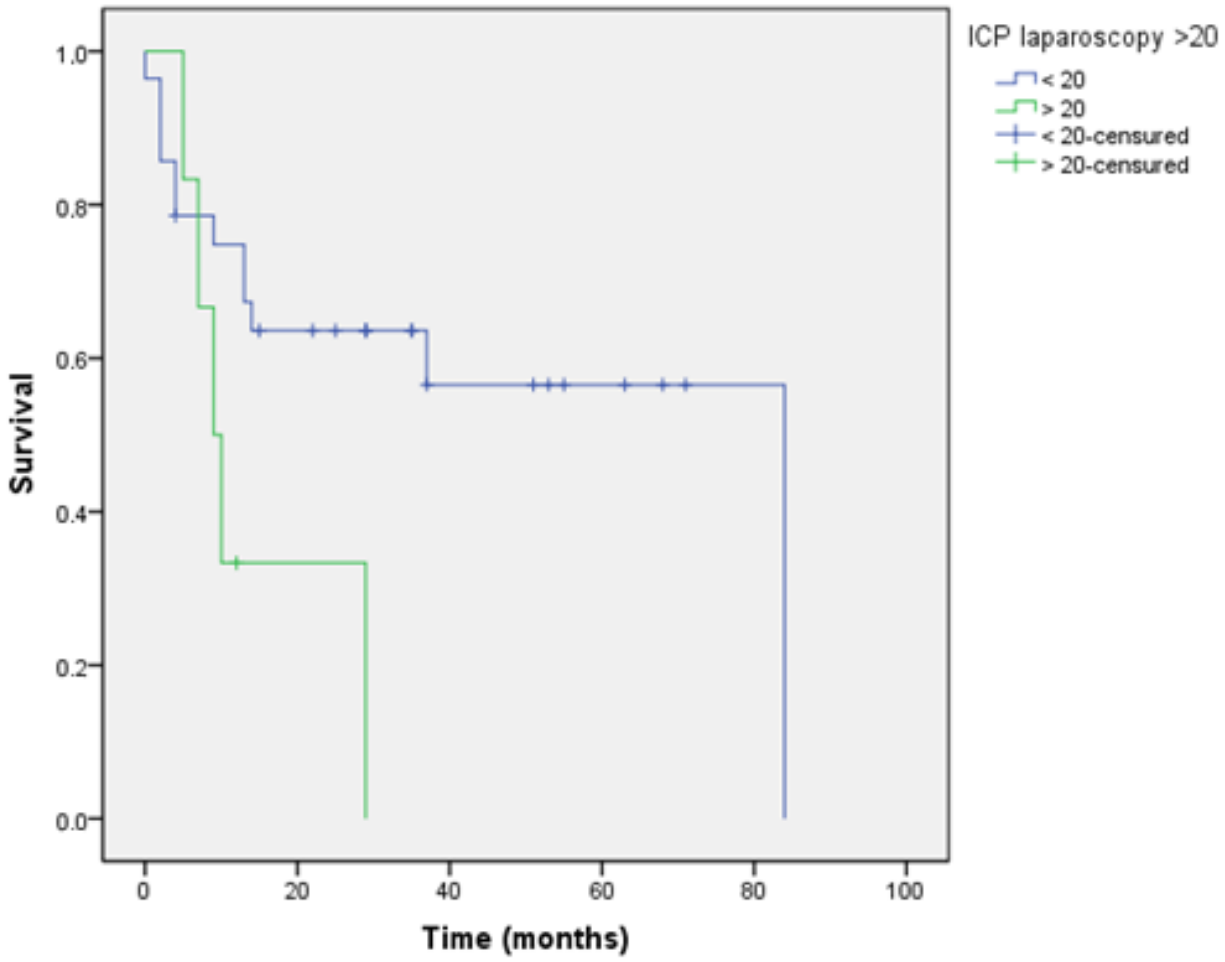

Figure 5. Survival PCI $>20$. 
Table 5. Survival PCI "cut off" 10.

\begin{tabular}{ccc}
\hline & Mean Overall Survival (Months) & $\mathbf{9 5 \%}$ CI \\
\hline PCI LPS $<10$ & 69.27 & $46.66-91.89$ \\
PCI LPS $\geq 10$ & 20.22 & $13.82-26.61$ \\
\hline
\end{tabular}

PCI LPS: Laparoscopic Peritoneal Carcinomatosis Index.

Table 6. Survival PCI "cut off" 20.

\begin{tabular}{ccc}
\hline & Mean Overall Survival (Months) & $\mathbf{9 5 \%}$ CI \\
\hline PCI LPS $<20$ & 52.44 & $37.35-67.54$ \\
PCI LPS $\geq 20$ & 14.38 & $5.76-23.90$ \\
\hline
\end{tabular}

PCI LPS: Laparoscopic Peritoneal Carcinomatosis Index.

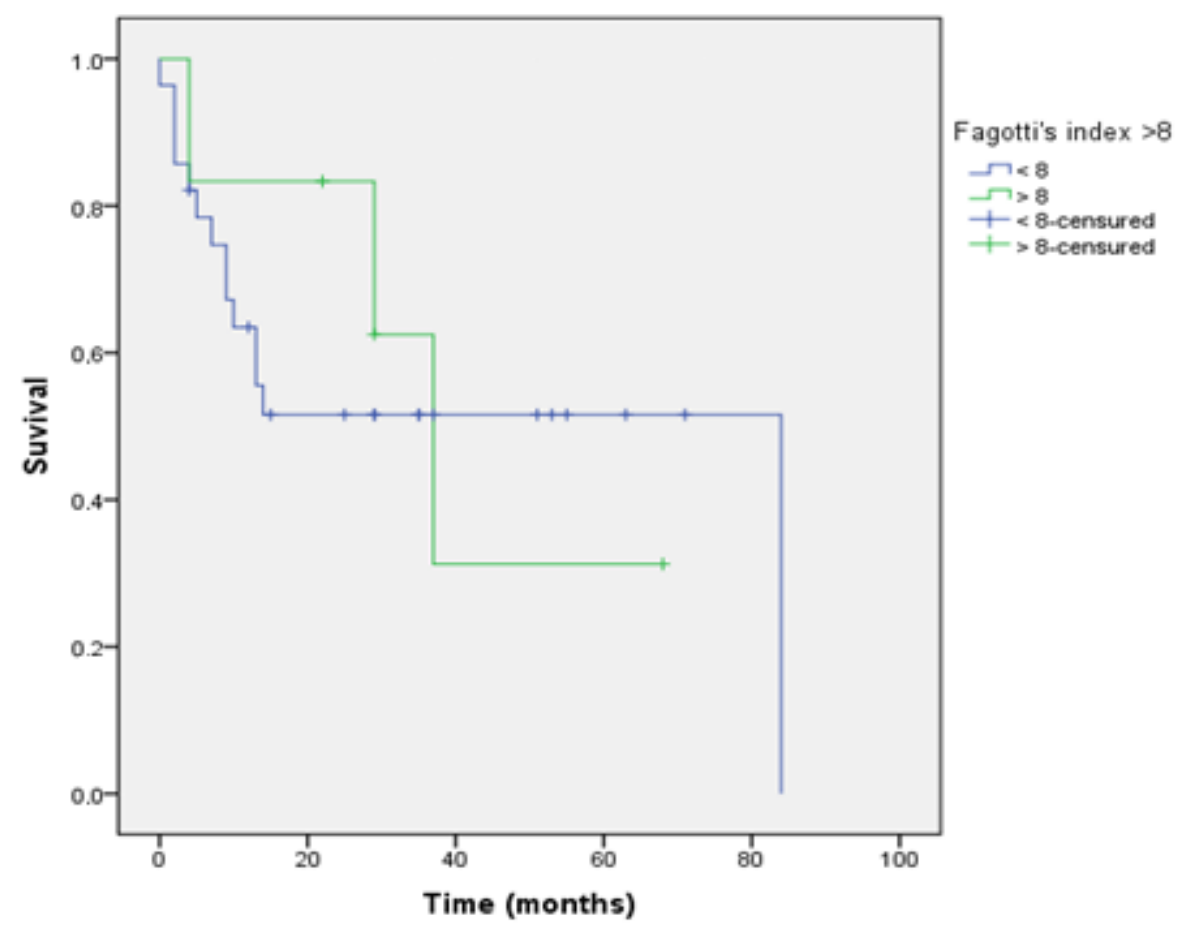

Figure 6. Survival Fagotti's Index.

Table 7. Survival Fagotti "cut off" 8.

\begin{tabular}{ccc}
\hline & Mean Overall Survival (Months) & 95\% CI \\
\hline Fagotti $<8$ & 47.76 & $31.54-61.97$ \\
Fagotti $\geq 8$ & 39.52 & $19.25-59.71$ \\
\hline
\end{tabular}

To determine the risk of incomplete debulking, the relative risk was calculated for the scores described by the "cut off."

A laparoscopic-PCI > 20 was a risk factor for incomplete debulking and unnecessary laparotomy RR 3.5 95\% CI (10.44-11.734).

This association was not evidenced with PCI cut-off of 10, nor with the Fagotti score.

\section{Discussion}

The present study shows that the peritoneal carcinomatosis index described by Sugarbaker for colorectal cancer is a valid diagnostic test that provides prognostic value in evaluating peritoneal carcinomatosis of gynaecological origin.

Patients with a PCI greater than 20 showed a decrease of 37.61 months in overall survival $[6,14]$. 
The results obtained in our sample for Fagotti's index in specificity and positive predictive value present values below those described in their study, showing an AUC of 0.66 , also lower than that obtained in the external validation of Brun et al. and Chéreau, which was 0.74 and 0.69 , respectively $[6,8,10-12]$ These findings are probably a consequence of the suboptimal cytoreduction rate since in Fagotti's validation study, it was $32.8 \%$, while in this sample, the rate is $20.6 \%$ [10]

The best diagnostic test to determine the eligible patients for neoadjuvant treatment is the Sugarbaker's peritoneal cancer index, increasing diagnostic accuracy with the increase in the cut-off point.

This cut-off point should be established according to the complete debulking rate obtained in each working group [13].

One of the factors that influence overall survival is the volume of the disease.

The article by Llueca et al. $[5,6,13]$ shows that the subgroup of patients with PCI less than or equal to 10 presents better survival than a value above 20 . These findings are confirmed in our study with a decrease in survival as the peritoneal cancer index value increases. This prognostic impact is not observed in Fagotti's model because it is not a model that quantifies disease.

The gold standard for evaluating the burden of peritoneal carcinomatosis is laparotomy. In an article by Llueca et al. [5], two predictive models were compared; the accuracy of this evaluation increases with the laparotomic exploration $[9,15,16]$. However, due to the morbidity associated with this technique, it is not considered a diagnostic technique. [17]. However, the potential discrepancy between the PCI assessment by laparoscopy and laparotomy could interfere with the main objective of the study $[5,15,18]$.

Nevertheless, the objective of the study is to establish a better index to apply the laparoscopic method to identify with the least error the patients who would benefit from neoadjuvant therapy because complete debulking surgery is unlikely $[19,20]$.

One of the strengths of this study is the homogeneity of the sample. According to the same criteria for unresectability, all patients were evaluated and treated by the same surgical team with an established suboptimal cytoreduction rate (tumour residue greater than $1 \mathrm{~cm}$ ) of approximately $16 \%$.

Another noteworthy point of this study is to include patients with peritoneal carcinomatosis of endometrial origin. This subgroup has a worse prognosis as it is a tumour with less chemosensitivity than the ovary, and neoadjuvant treatment in this group is not standard clinical practice $[9,21]$.

The study's main limitation is a retrospective evaluation of the data. A selection bias was achieved when choosing the patients who underwent laparoscopy and later laparotomy. This fact implies that the surgical team has already selected the patients as probably resectable. This is the reason why there are few patients with PCI values greater than 20 and few patients with suboptimal surgery. Another limitation is the inclusion of patients undergoing neoadjuvant therapy (23.5\%). This patient usually has a lower burden of disease; however, when subjected to laparoscopy before surgery, they probably have a greater volume of disease greater than expected in the pre-surgical study [3,22].

One of the weakness of the study is the small sample size, but there is a lack of information in this field, and we could contribute to closing the gap.

It is necessary to carry out prospective studies with a greater number of patients to verify the results obtained in our evaluation.

\section{Conclusions}

Based on the data obtained in our study, it is possible to suggest that the best diagnostic method to classify patients with peritoneal cancer to indicate primary treatment is the peritoneal carcinomatosis index. It presents a better correlation of the extension of the disease and provides prognostic information. 
We consider this score could be adapted to the suboptimal cytoreduction rate of each surgical team, allowing the cut-off point to be established at a particular moment and evolving according to the results obtained by the surgical team.

Author Contributions: Conceptualization, M.T.C., A.S., J.G.-E., J.G.-A. and A.L.; data curation, M.T.C. and J.G.-E.; formal analysis, M.T.C., J.G.-E. and J.G.-A.; investigation, M.T.C. and A.L.; methodology, M.T.C. and A.L.; software, A.S.; supervision, J.G.-E., J.G.-A. and A.L.; visualization, J.G.-A.; writing—original draft, M.T.C.; writing—review and editing, M.T.C., A.S. and A.L. All authors have read and agreed to the published version of the manuscript.

Funding: This work received financial support from de Medtronic University Chair for Training and Surgical Research, University Jaume I (UJI), Castellón, Spain. The study is also supported by a research grant from ISCIII-FEDER (PI17/01945).

Institutional Review Board Statement: The study was conducted according to the guidelines of the Declaration of Helsinki, and approved by the Ethics Committee of University General Hospital of Castellon (HIP/CI version (3 June 2021)).

Informed Consent Statement: Informed consent was obtained from all subjects involved in the study.

Data Availability Statement: The data presented in this study are available on request from the corresponding author. The data are not publicly available due to privacity of the patients included in this article.

Acknowledgments: The authors would like to thank Begoña Bellés-Fortuño for editing and proofreading the manuscript. *MUAPOS working group (Multidisciplinary Unit of Abdominal Pelvic Oncology Surgery): Gomez-Quiles L., Játiva R., Cebrian G., Bosso, V., Villarin, A., Maiocchi, K., Delgado-Barriga K., Rodrigo-Aliaga M., Ruiz N., Herrero C., Frances, A., Beato I., Ferrer C., Aracil J.P., Boldo E., Boldo A. and Adell R.

Conflicts of Interest: The authors declare no conflict of interest.

\section{Appendix A}

Index Carcinomatosis Peritoneal:

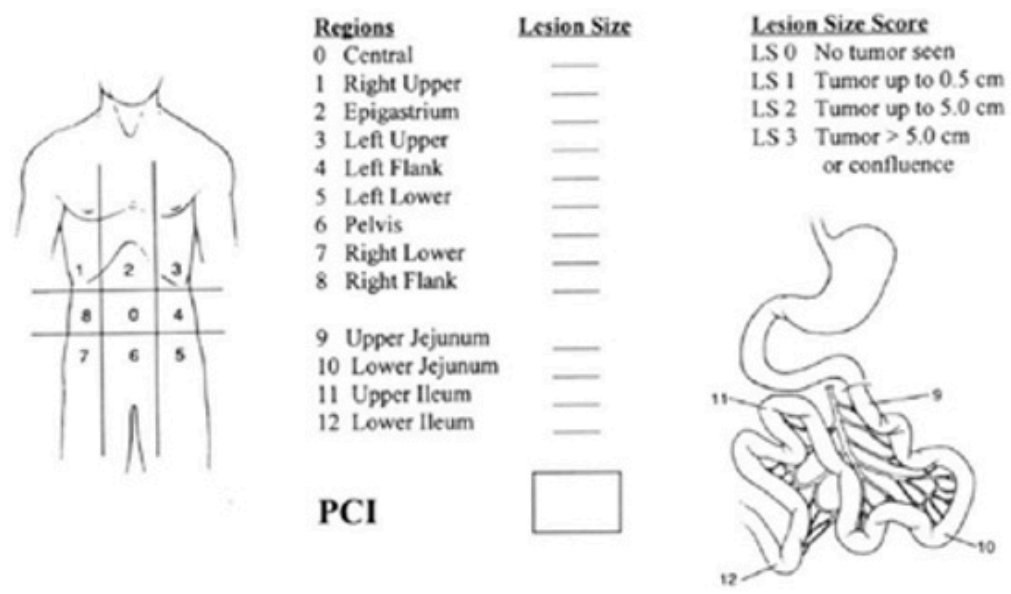

Figure A1. Sugarbaker Index Carcinomatosis Peritoneal. 
Table A1. Fagotti's Index.

\begin{tabular}{ccc}
\hline Fagotti's Index & $\mathbf{0}$ & $\mathbf{2}$ \\
\hline Omental cake & & \\
Peritoneal carcinosis & & \\
Diaphragmatic carcinosis & \\
Mesenteric retraction & \\
Bowel infiltration & \\
Stomach infiltration & \\
Liver metastases & \\
\hline
\end{tabular}

\section{References}

1. Llueca, A.; Serra, A.; Herraiz, J.L.; Rivadulla, I.; Gomez-Quiles, L.; Gilabert-Estelles, J.; Escrig, J. Peritoneal carcinomatosis index as a predictor of diaphragmatic involvement in stage III and IV ovarian cancer. OncoTargets Ther. 2018, 11, 2771-2777. [CrossRef]

2. Van De Vrie, R.; Rutten, M.J.; Asseler, J.D.; Leeflang, M.M.; Kenter, G.G.; Mol, B.W.; Buist, M. Laparoscopy for diagnosing resectability of disease in women with advanced ovarian cancer. Cochrane Database Syst Rev. 2019. [CrossRef] [PubMed]

3. Zivanovic, O.; Sima, C.S.; Iasonos, A.; Hoskins, W.J.; Pingle, P.R.; Leitao, M.M., Jr.; Sonoda, Y.; Abu-Rustum, N.R.; Barakat, R.R.; Chi, D.S. The effect of primary cytoreduction on outcomes of patients with FIGO stage IIIC ovarian cancer stratified by the initial tumor burden in the upper abdomen cephalad to the greater omentum. Gynecol. Oncol. 2010, 116, 351-357. [CrossRef]

4. "SEGO Oncoguide": Epithelial Cancer of the Ovary, Tube and Peritoneum 2014. Clinical Practice Guidelines in Gynecological and Breast Cancer. October 2014, Volume 2. Available online: http:/ /www.svgo.es/sites/default/files/OvarioOncoguia2008.pdf (accessed on 20 March 2021).

5. Llueca, A.; Serra, A.; Delgado, K.; Maiocchi, K.; Jativa, R.; Gomez, L.; Escrig, J. A radiologic-laparoscopic model to predict suboptimal (or complete and optimal) debulking surgery in advanced ovarian cancer: A pilot study. Int. J. Women's Health 2019, 11, 333-342. [CrossRef]

6. $\quad$ Llueca, A.; Escrig, J.; Serra-Rubert, A.; Gomez-Quiles, L.; Rivadulla, I.; Játiva-Porcar, R.; Moreno-Clarí, E.; Montañés-Pauls, B.; Granel-Villach, L.; Villegas-Cánovas, C.; et al. Prognostic value of peritoneal cancer index in primary advanced ovarian cancer. Eur. J. Surg. Oncol. 2018, 44, 163-169. [CrossRef] [PubMed]

7. Fagotti, A.; Ferrandina, G.; Fanfani, F.; Ercoli, A.; Lorusso, D.; Rossi, M.; Scambia, G. A laparoscopy-based score to predict surgical outcome in patients with advanced ovarian carcinoma: A pilot study. Ann. Surg. Oncol. 2006, 13, 1156-1161. [CrossRef]

8. Greggi, S.; Falcone, F.; Scaffa, C.; du Bois, A.; Samartzis, E.P.; Pujade-Lauraine, E.; Cibula, D.; Mądry, R.; Korach, J.; Gungorduk, K.; et al. Evaluation of surgical resection in advanced ovarian, fallopian tube, and primary peritoneal cancer: Laparoscopic assessment. A European Network of Gynaecological Oncology Trial (ENGOT) group survey. Int. J. Gynecol. Cancer 2020, 30, 819-824. [CrossRef] [PubMed]

9. Varnoux, C.; Huchon, C.; Bats, A.S.; Bensaid, C.; Achouri, A.; Nos, C.; Lécuru, F. Diagnostic accuracy on hand assisted laparoscopy in predicting resecability of peritoneal carcinomatosis from gynecological malignancies. EJSO 2013, 39, 774-779. [CrossRef] [PubMed]

10. Brun, J.L.; Rouzier, R.; Uzan, S.; Daraï, E. External validation of a laparoscopic-based score to evaluate resectability of advanced ovarian cancers: Clues for a simplified score. Gynecol. Oncol. 2008, 110, 354-359. [CrossRef] [PubMed]

11. Chéreau, E.; Ballester, M.; Selle, F.; Cortez, A.; Daraï, E.; Rouzier, R. Comparison of peritoneal carcinomatosis scoring methods in predicting resectability and prognosis in advanced ovarian cancer. Am. J. Obstet. Gynecol. 2010, 202, 178.e1-178.e10. [CrossRef] [PubMed]

12. Fagotti, A.; Fanfani, F.; Vizzielli, G.; Gallotta, V.; Ercoli, A.; Paglia, A.; Costantini, B.; Vigliotta, M.; Scambia, G.; Ferrandina, G. Should laparoscopy be included in the work-up of advanced ovarian cancer patients attempting interval debulking surgery? Gynecol. Oncol. 2010, 116, 72-77. [CrossRef]

13. Llueca, A.; Serra, A.; Rivadulla, I.; Gomez, L.; Escrig, J. and MUAPOS working group (Multidisciplinary Unit of Abdomino -Pelvic Surgery). Prediction of suboptimal cytoreductive surgery in patients with advanced ovarian cancer based on preoperative and intraoperative determination of the peritoneal carcinomatosis index. World J. Surg. Oncol. 2018, 16, 1-7.

14. Rosendahl, M.; Harter, P.; Bjørn, S.F.; Høgdall, C. Specific regions, rather than the entire peritoneal carcinosis index, are predictive of complete resection and survival in advanced epithelial ovarian cancer. Int. J. Gynecol. Cancer 2018, 28, 316-322. [CrossRef]

15. Fagotti, A.; Vizzielli, G.; De Iaco, P.; Surico, D.; Buda, A.; Mandato, V.D.; Petruzzelli, F.; Ghezzi, F.; Garzarelli, S.; Mereu, L.; et al. A multicentric trial (Olympia-MITO 13) on the accuracy of laparoscopic to assess peritoneal spread in ovarian cancer. Am. J. Obstet. Gynecol. 2013, 209, 462.e1-462.e11. [CrossRef]

16. Bhatt, A.; Rouseet, P.; Benzerdjeb, N.; Kammar, P.; Mehta, S.; Parikh, L.; Goswami, G.; Shaikh, S.; Kepenekian, V.; Passot, G.; et al. Prospective correlation of the radiological, surgical and pathological findings in patients undergoing cytorreductive surgery for colorectal peritoneal metastases: Implications for the preoperative estimation of the peritoneal cancer index. Colorrectal Disease 2020, 22, 2123-2132. [CrossRef] 
17. Rutten, M.J.; Van Meurs, H.S.; Van De Vrie, R.; Naaktgeboren, C.A.; Fons, G.; Opmeer, B.C.; Spijkerboer, A.; Bossuyt, P.M.; Kenter, G.G.; Buist, M.R.; et al. Laparoscopy to predict the result of primary cytoreductive surgery in patients with advanced ovarian cancer: A randomised controlled trial. Clin. Oncol. 2016, 35, 613-627. [CrossRef] [PubMed]

18. Muallem, M.Z.; Sehouli, J.; Richter, R.; Babayeva, A.; Gasimli, K.; Parashkevova, A. Pre-operative serum Ca 125, peritoneal cancer index and intra-operative mapping score as predictors of surgical results in primary epithelial ovarian cancer. Int. J. Gynecol. Cancer 2020, 30, 62-66. [CrossRef] [PubMed]

19. Aletti, G.D.; Eisenhauer, E.L.; Santillan, A.; Axtell, A.; Aletti, G.; Holschneider, C.; Chi, D.S.; Bristow, R.E.; Cliby, W.A. Identification of patient groups at highest risk from traditional approach to ovarian cancer treatment. Gynecol. Oncol. 2011, 120, 23-28. [CrossRef] [PubMed]

20. Javadi, S.; Ganeshan, D.M.; Qayyum, A.; Iyer, R.B.; Bhosale, P. Ovarian cancer, the revised FIGO staging system, and the role of imaging. Am. J. Roentgenol. 2016, 206, 1351-1360. [CrossRef] [PubMed]

21. Landrum, L.M.; Moore, K.N.; Myers, T.K.; Lanneau, G.S.; Walker, J.L.; Gold, M.A. Stage IVB endometrial cancer: Does applying an ovarian cancer treatment paradigm result in similar outcomes: A case-control analysis. Gynecol. Oncol. 2009, 112, 337-341. [CrossRef] [PubMed]

22. Prat, J.; FIGO Committee on Gynecology Oncology. FIGO's staging classification for cancer of the ovary, fallopian tube, and peritoneum: Abridged republication. J. Gynecol. Oncol. 2015, 26, 87-89. [CrossRef] [PubMed] 